\title{
Incorporating single port video-assisted thoracic surgery training into regular thoracic surgery training programs
}

\author{
John K. C. Tam \\ Department of Surgery, National University of Singapore, Singapore \\ Correspondence to: John K. C. Tam. Department of Surgery, National University of Singapore, Singapore 119228. Email: john_tam@nuhs.edu.sg.
}

Received: 25 May 2018; Accepted: 19 July 2018; Published: 14 August 2018.

doi: 10.21037 /jovs.2018.07.22

View this article at: http://dx.doi.org/10.21037/jovs.2018.07.22

\section{My background in surgical education}

Between 2006 to 2011, I was the sole dedicated consultant thoracic surgeon serving 3 hospitals in Singapore with a combined total of $>2,500$ beds, and a catchment population of $>2$ million people. At that same time, I served as both the undergraduate and postgraduate director of surgical education at the National University of Singapore and the National University Hospital of Singapore. Since 2012, I was the Head of the Division of Thoracic Surgery and Lung Surgery Centre in the National University Health System, Singapore, and also the director of the Advanced Thoracic Surgery Fellowship Program in our institution. Over the past decade, I have directly trained more than 8 consultant-level thoracic surgeons in Singapore and overseas, and multiple surgical trainees in thoracic surgery.

\section{Single port video-assisted thoracic surgery (VATS) training}

The word "incorporate" originates from the Latin word "corpus", which means take in or contain something as part of a whole, and combine something into one substance and one body. As such, successful uniportal video-assisted thoracic surgery (UVATS) training must be rooted within a good UVATS program.

UVATS training just like any good surgical training should be structured, progressive, competency-based, patient safety oriented, and graduating from easy to difficult procedures. Entrustable Professional Activities (EPAs) and Procedural Based Assessment (PBAs) are tools that keep surgical training on the right path. It is important to recognize that there are multiple pathways to skill acquisition that can lead to eventual skill mastery.

\section{Hierarchy of skills}

There are a variety of procedures within the full spectrum of thoracic surgery that require various levels of skills. This can range from UVATS incision making, thoracoscopic visualization and exploration, pneumothorax surgery, decortication, lobectomy, segmentectomy, and mediastinal surgery. For major lung resections, the trainee can progress from dissecting various key structures, which include the inferior pulmonary ligament, pulmonary vein, pulmonary artery, bronchus, fissure, and mediastinal lymph nodes. Each structure requires a gamut of thoracoscopic skills, including dissection, stapling, suturing and suture ligation, vascular control, and anastomosis creation. Over the training period, further advanced skills can be acquired, including how to treat complications such as pulmonary artery injury or thoracic duct leak, fissure-less approach with retrograde dissection, UVATS surgery with incomplete lung isolation and ventilated lung, and more challenging conditions such as post chemotherapy and radiotherapy, or post tuberculosis (TB) scarring.

\section{Avenues of UVATS skill acquisition}

There are many avenues where UVATS skills can be acquired, each of them has important merits for UVATS trainees at different stages of their learning. Practicing in the dry lab or wet lab is an important venue for beginners. Attending conferences, symposiums and workshops allow trainees to be exposed to showcasing of expert techniques. 


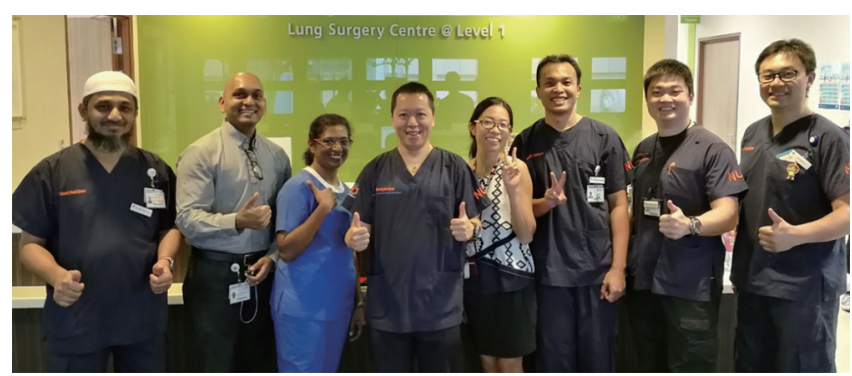

Figure 1 Our team at the Lung Surgery Centre in the National University Hospital, Singapore in 2017.

Formal residency and fellowship within an excellent UVATS training program is a key avenue of skill acquisition for young learners. Gradual progression to UVATS in a "live" surgery setting either independently or with proctorship may be an important avenue for more established thoracic surgeons in practice while upholding patient safety as a paramount priority.

\section{Key pathways of UVATS skill acquisition}

There are two key pathways for surgeons to become proficient in UVATS. The first pathway is to gradually move from open surgery to multiportal VATS and eventually to uniportal VATS. Bridging techniques for this pathway are hybrid surgery (open with thoracoscopic assist) and multiport approach while reducing ports one by one until there is only uniport. This can be considered as the "old" way of progression for more established thoracic surgeons already in practice.

Direct UVATS is the second and better pathway for UVATS skill acquisition. This is especially useful and practical for the new generation of surgeons that can benefit from direct mentorship. Within a good UVATS residency and fellowship training program, these young surgeons can benefit from direct immersion in the right environment, and can enjoy proctorship, mentorship and leadership of established UVATS surgeons.

\section{Team approach in UVATS training}

The director and trainer of the UVATS program have the important roles of grooming, teaching, supporting, assisting, and team building. He/she has to be an expert UVATS surgeon who looks after the well-being and progress of the surgical team members and the patients, while also manage and enable other associated teams including anesthesia, respirology, oncology, radiology, pathology, nursing, allied health, and administrative teams. UVATS program is a team effort, and good team leadership is indispensable to its success. Once a surgeon has acquired a certain skill set, he or she is encouraged and expected to take the lead and pass the skill onwards to other learners. In such a way, learning becomes shared and is passed on by paying it forward to the next generation.

\section{Conclusions}

Successful UVATS training must be rooted in a successful UVATS program. The best pathway to UVATS skill acquisition is by embedding trainees into the UVATS program through direct immersion and structured progression within a safety-oriented learning environment. Direct UVATS approach is the preferred way of training new UVATS surgeons. Team building is crucial to the success of the UVATS training program. Teaching and mentorship of the next generation of UVATS surgeons are well worth the time and effort (Figure 1).

\section{Acknowledgments}

Funding: None.

\section{Footnote}

Provenance and Peer Review: This article was commissioned by the Guest Editor (Jose Luis Danguilan) for the series "Dedicated to the 6th Asian Single-port VATS Symposium 2018" published in fournal of Visualized Surgery. The article has undergone external peer review.

Conflicts of Interest: The author has completed the ICMJE uniform disclosure form (available at http://dx.doi. org/10.21037/jovs.2018.07.22). The series "Dedicated to the 6th Asian Single-port VATS Symposium 2018" was commissioned by the editorial office without any funding or sponsorship. The author has no other conflicts of interest to declare.

Ethical Statement: The author is accountable for all aspects of the work in ensuring that questions related to the accuracy or integrity of any part of the work are appropriately investigated and resolved.

Open Access Statement: This is an Open Access article 
distributed in accordance with the Creative Commons Attribution-NonCommercial-NoDerivs 4.0 International License (CC BY-NC-ND 4.0), which permits the noncommercial replication and distribution of the article with

doi: 10.21037/jovs.2018.07.22

Cite this article as: Tam JK. Incorporating single port videoassisted thoracic surgery training into regular thoracic surgery training programs. J Vis Surg 2018;4:171. the strict proviso that no changes or edits are made and the original work is properly cited (including links to both the formal publication through the relevant DOI and the license). See: https://creativecommons.org/licenses/by-nc-nd/4.0/. 